\title{
Incorporating environmental uncertainty in fire spread modelling
}

\author{
H.H. Zazali, ${ }^{\mathrm{a}}{ }^{\text {I.N. }}$. Towers ${ }^{\mathrm{a}}$ and J.J. Sharples ${ }^{\mathrm{a}}$

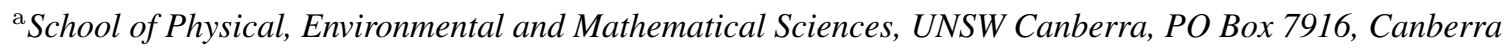 \\ 2610, Australia \\ Email: $\underline{\text { h.zazali@student.adfa.edu.au }}$
}

\begin{abstract}
Bushfire simulators provide fire managers with a means of estimating the likely progression of a fire across a landscape. However, the accuracy of bushfire simulators is confounded by the various forms of uncertainty or variability that are found in the data that informs the simulation. Indeed, given the sensitivity of fire spread models to environmental factors such as wind speed and direction, variability in environmental inputs can result in large differences between the simulated and actual progression of a bushfire. To address these issues recent efforts have been directed at incorporating environmental uncertainty through ensemble fire spread modelling approaches. The approaches currently being explored employ a deterministic fire spread model, which is run multiple time using inputs drawn from a probability distribution that represents the variability in the data. While this approach does provide more information on the range of different fire spread scenarios that may arise, there are doubts about whether such an approach provides a faithful representation of the way environmental variability affects fire spread.
\end{abstract}

In this paper we consider an alternate approach to incorporating environmental uncertainty in fire spread models. In particular, we consider the problem in terms of stochastic calculus, which incorporates stochasticity in the environmental variables in an intrinsic way. In this initial preliminary work we couple a deterministic fire spread model with simple stochastic differential equations for wind speed and direction, which are considered as state variables.

The results arising from the stochastic model are compared with those arising from a deterministic ensemble approach, in which the variability in wind speed and direction are chosen randomly at each time step of a deterministic model run. In this approach the wind speed and direction are not considered as state variables, but are treated extrinsically as random input variables.

Both approaches were implemented 5000 times to develop an overall picture of the distribution of fire propagation scenarios accommodated by each method. It was found that the stochastic approach resulted in a broader range of possible fire propagation scenarios compared to the ensemble approach. In particular, the stochastic approach indicated that the region impacted by fire with a $99 \%$ likelihood extended to $18.2 \%$ further downwind than what would be expected using the ensemble approach.

The findings of this study indicate that the way environmental uncertainty is incorporated in fire propagation models can make a considerable difference to their ensuing predictions. This has ramifications for the development of probabilistic approaches to fire impact and risk assessment; in particular it suggests that current approaches may underestimate the likely propagation of a bushfire.

Keywords: Stochastic calculus, fire spread, bushfire, environmental uncertainty, deterministic process 
H H. Zazali, et al. Incorporating environmental uncertainty in fire spread modelling

\section{INTRODUCTION}

Bushfire spread models are of great importance in assisting the decision making process during emergencies. The continued development and improvement of such models is therefore of keen interest to a range of stakeholders from the fire services to wildlife agencies. In Australia itself, a variety of bushfire simulation models exist, which, depending on the model's approach and underlying assumptions are used for different environmental situations. One such model is the McArthur's Forest Fire Danger Index (FFDI) by A. G. McArthur, which was among the earliest models developed to forecast Australian fire behaviour in eucalypt forests (McArthur, 1967; Noble et al., 1980). Due to the effectiveness (and early development) of the FFDI, this model is still in use.

The progress in fire behaviour studies has seen improvements in the breadth of environmental factors that models can incorporate. In turn, models are claimed to have greater predicative power and areas of applicability. However, the sheer complexity of the time-varying parameter space of a bushfire, coupled with sometimes significant uncertainty in the values of said parameters, has led many researchers to consider so-called ensemble methods. Ensemble methods can be seen as a first step away from fully deterministic models, which by their nature assume no uncertainty in inputs.

The ensemble approach can be seen in frameworks such as the Fire Impact and Risk Evaluation Decision Support Tool (FireDST) (French et al., 2013) and the Simulation Analysis-based Risk Evaluation (SABRE) Fire framework (Twomey and Sturgess, 2016). Broadly speaking, a typical ensemble approach will involve using input parameter values sampled from a particular probability distribution. The simulation is then conducted via a deterministic model to produce a single realisation of fire spread over a given time frame. The process is repeated a multitude of times, each time sampling new input parameters. Some method of averaging the realisations is utilised to calculate the typical or most probable fire spread over the course of the simulation, but the full range of data produced also provides information on 'worst case' scenarios, which can be of more use to fire managers.

Due to the turbulent nature of the atmospheric boundary layer, variations in vegetation and the intricacies of the actual terrain, the broad-scale fire spread model inputs should only be considered as valid in some mean sense. There will be significant spatio-temporal variation in these input parameters, which can affect the spread of a fire. Incorporating such inputs makes the development process of a fire simulator model more complex. There are numerous efforts to achieve the degree of accuracy in the development of fire prediction models, depending on the model's capability in a given situation, the validity of the model's relationship and the reliability of the model input data (Alexander and Cruz, 2013).

Incorporating environmental elements into a bushfire spread model is an obvious necessity even if the goal is to only study the phenomena in qualitative terms. However, how said elements are modelled and the choice of simulation procedure will have significant impact on the overall bushfire spread model's utility. Ensemble methods are attempting to address the uncertainty in the input parameters. As Alexander and Cruz (2013) state, the foremost issue for the bushfire modeller is the accuracy of the input parameters, as any deterministic model will assume they are certain and unchanging. Both Albini (1976) and Zhang et al. (2016) have reported that inherent error in the input data to a prediction model negatively influences overall performances.

For instance, a simulation analysis using FARSITE was conducted by Fujioka (2001). The study showed significant errors in the calculated fire perimeter after only 12 minutes when compared to actual historical fire data. Fujioka believed the errors were due to the varying environmental conditions that FARSITE was unable to capture. Similar errors were found in the Canadian fire model, PROMETHEUs (Johnston et al., 2005). This deterministic model delivers outputs based on the assumed accuracy and unchanging nature of the input parameters, which will lead to inaccurate results when the fire being simulated evolves in a highly variable environment. Therefore, the output of deterministic fire spread models may produce realistic average patterns, but are unable to incorporate stochastic variability of the environment and deal with imperfect input parameters (Boychuk et al., 2009).

While ensemble methods acknowledge the uncertainty in fire spread modelling inputs to a certain degree, they make no attempt to address the spatio-temporal fluctuation in environmental parameters. The complexity of a bushfire interacting with its environment leads to an intrinsic randomness in parameters. As an extrinsic methodology, ensemble approaches cannot describe random dynamical effects. Cruz (2010) points out that incorporating stochastic analysis in fire spread models would be more valuable in obtaining error bounds and probability-based outcomes in the prediction models. Hajian et al. (2016) agree that stochastic analysis has advantages over the deterministic approach, while Han and Braun (2014) go as far as stating that a realistic 
fire spread model, based on rate of spread, should have a stochastic component.

This paper will focus on using stochastic calculus as a basic fire spread model development tool, incorporating wind as a state-variable, which will be allowed to vary stochastically throughout the simulation process. We compare the results of this fully stochastic fire spread model to those produced by an ensemble of deterministic calculations. The examples considered in this paper illustrate significant differences between the two approaches. The implications for fire management will be demonstrated in the following sections.

\section{Elliptical Fire PROPAgATion}

In two dimensional Euclidean space, the perimeter of a bushfire at any point in time is defined by a simple closed curve. Suppose that a fire line has evolved at time $t$ defined by a curve $\gamma(\theta)$ and the position vector can be written as $\mathbf{x}(\theta, t)=(x(\theta, t), y(\theta, t))$. To determine a new fire front at $t+\Delta t$, a mathematical approach based on Huygens' principle (Anderson et al., 1982) is used. This concept assumes that for a fire originating from a point ignition, and evolving under the influence of a constant and spatially uniform wind, the closed curve that the fire line makes at any particular time is an ellipse. Then given an arbitrary fire line described by the curve $\gamma(\theta)$ at time $t$, the new fire line at time $t+\Delta t$ is determined by assuming that each point of $\gamma(\theta)$ acts as a point ignition to a family of elliptic frontlets. The dimension and orientation of each of the elliptic frontlets is determined by wind conditions, and the new fire line at $t+\Delta t$ is defined as the envelope of the family of elliptic frontlets.

Assuming that the point ignition occurs at the origin, and that the wind is blowing in the direction of the positive $x$-axis - so that the wind vector is $\underset{\sim}{\mathbf{w}}=(w, 0)$, the basic spread of a wind-driven fire can be described by the following equations for the position of the fire line

$$
\begin{aligned}
& x(\theta, t)=R_{0} t(f \cos \theta+g), \\
& y(\theta, t)=R_{0} \text { th } \sin \theta, \quad \theta \in[0,2 \pi) .
\end{aligned}
$$

where, $R_{0}$ is the basic rate of spread expressed in kilometres per hour $\left(\mathrm{km} \mathrm{h}^{-1}\right), t$ is in hours (h) and $f, g$, and $h$ are wind speed dependent parameters that define the dimensions of the ellipse.

In the following we assume that the flanks of the fire are not influenced by the wind, so that the flank fire rate of spread is equal to $R_{0}$. We also assume that the back fire behaves as if it was a head fire burning under the influence of a 'negative' wind. While these assumptions may not be entirely justified, they result in reasonable model behaviour, and have no real bearing on the results of our comparative analysis. Following from these assumptions we may write the elliptic shape parameters $f, g$, and $h$ in terms of the McArthur Mark 5 Forest Fire Danger Rating System (Noble et al., 1980) as

$$
\begin{aligned}
& f(w)=\cosh (\alpha w), \\
& g(w)=\sinh (\alpha w), \\
& h(w)=1 .
\end{aligned}
$$

where $\alpha=0.0234\left(\mathrm{~h} \mathrm{~km}^{-1}\right)$ and wind speed has units of $\mathrm{km} \mathrm{h}^{-1}$.

Equivalently, the fire front can be described as a normal flow (Roberts, 1993):

$$
\frac{\partial}{\partial t} \underset{\sim}{\mathbf{X}}(s, t)=F(\underset{\sim}{\mathbf{X}}(s, t), \underset{\sim}{\mathbf{w}}) \underset{\sim}{\hat{\mathbf{n}}}, \underset{\sim}{\mathbf{X}}(s, 0)=\gamma_{0}(s)
$$

with

$$
F(\underset{\sim}{\mathbf{X}}, \underset{\sim}{\mathbf{w}})=R_{0} g(\underset{\sim}{\hat{\mathbf{n}}} \cdot \underset{\sim}{\hat{\mathbf{w}}})+R_{0} \sqrt{h^{2}+\left(f^{2}-h^{2}\right)(\underset{\sim}{\hat{\mathbf{n}}} \cdot \underset{\sim}{\hat{\mathbf{w}}})^{2}}
$$

where $\underset{\sim}{\hat{\mathbf{n}}}$ is the unit normal vector to the curve $\underset{\sim}{\mathbf{X}}(s, t)$.

\subsection{Ensemble approach}

Equations (3) and (4) are used to model the spread of a fire under variable wind conditions. We assume that the wind remains constant for a time interval $\delta t$. For each $\delta t$ a new wind vector $\mathbf{w}$ is selected. Our approach to the ensemble methodology is different in that the environmental parameter (wind) is not fixed throughout a 
given realisation. Specifically, the wind speed and direction are selected at each $\delta t$ from a multivariate normal distribution.

In our particular simulations we used an initial wind vector $\underset{\sim}{\mathbf{w}}=(20,0)$. For every unit of time increment $\delta t$ of 1 minute, $\mathbf{w}$ was set by randomly selecting the wind speed from a normal distribution with $\mu=20 \mathrm{~km} \mathrm{~h}^{-1}$ and $\sigma=5 \mathrm{~km} \mathrm{~h}^{-1}$ and randomly selecting the wind direction from a normal distribution with $\mu=0^{\circ}$ and $\sigma=10^{\circ}$. Fire lines were evolved for 1 hour and 5000 realisations were computed allowing us to build up confidence envelopes for the spread of the fire under the variable wind conditions. We further investigated the variability in the ensemble approach by incorporating an additive noise in equation (3). However, this approach only showed minimal differences compared to the current method.

\subsection{Stochastic model}

We construct a stochastic bushfire spread model by treating wind as a stochastic state variable rather than as a parameter. We couple to equations (3) and (4) expressions for wind speed and wind angle

$$
\begin{aligned}
d w & =\delta_{1} d W_{1} \\
d \phi & =\delta_{2} d W_{2}
\end{aligned}
$$

where $\underset{\sim}{\mathbf{w}}=w(\cos \phi, \sin \phi)$, with $w=20 \mathrm{~km} \mathrm{~h}^{-1}$, and $d W_{1}$ and $d W_{2}$ represent Wiener processes (Øksendal, 1998). The magnitudes of the noise are defined by $\delta_{1}=5$ and $\delta_{2}=\frac{\pi}{18}$, respectively.

\section{Results}

Both the ensemble and the stochastic model were solved numerically using a front-tracking paradigm (Tryggvason et al., 2001). The initial condition for all simulations was circle centred at the origin of radius $0.5 \times 10^{-3}$ metres. The curve was discretised into $n=500$ points. The deterministic ensembles were generated by solving equation (3) with a standard adaptive Runge-Kutta scheme (Shampine and Reichelt, 1997). The stochastic model of equations (3) and (5) were solved using an improved Euler scheme (Roberts, 2012). After 5000 realisations of each methodology, we obtained clear comparison results between deterministic and stochastic processes. The following figures show the fire lines after 1 hour, spreading under the influence of a constant wind vector or a wind which varies stochastically.

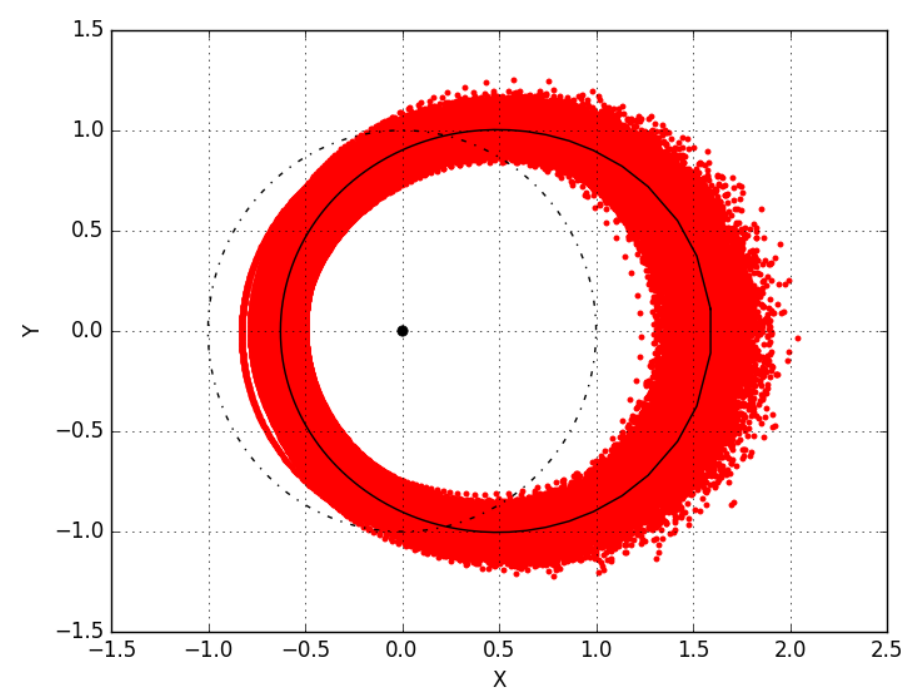

Figure 1. The extent of a bushfire, started at the origin, after 1 hour of evolution with $R_{0}=1$ in all cases. The dotted circle is the extent of the fire when there is no wind. The solid line is the fire line with a constant westerly wind. The red dots are the 5000 realisations of the stochastic model (equations 3, 4, 5).

Simulation analyses from both methods, ensemble and stochastic systems are shown in Figure 1 and Figure 2. Figure 1 shows 5000 realisations of fire line driven by a stochastic wind (red dots) starting from an initial "spot fire" at the origin (solid black dot) after one hour. As a comparison, the extent of fire spread at the same 
time with a constant wind $\mathbf{w}=(20,0)$ is shown by the solid line, with the spread under no wind (i.e. $\mathbf{w}=\mathbf{0}$ ) shown by the dotted black line. In all cases $R_{0}=1$. The stochastic wind clearly gives a much broader range of fire lines after the 1 hour time frame than the constant westerly wind.

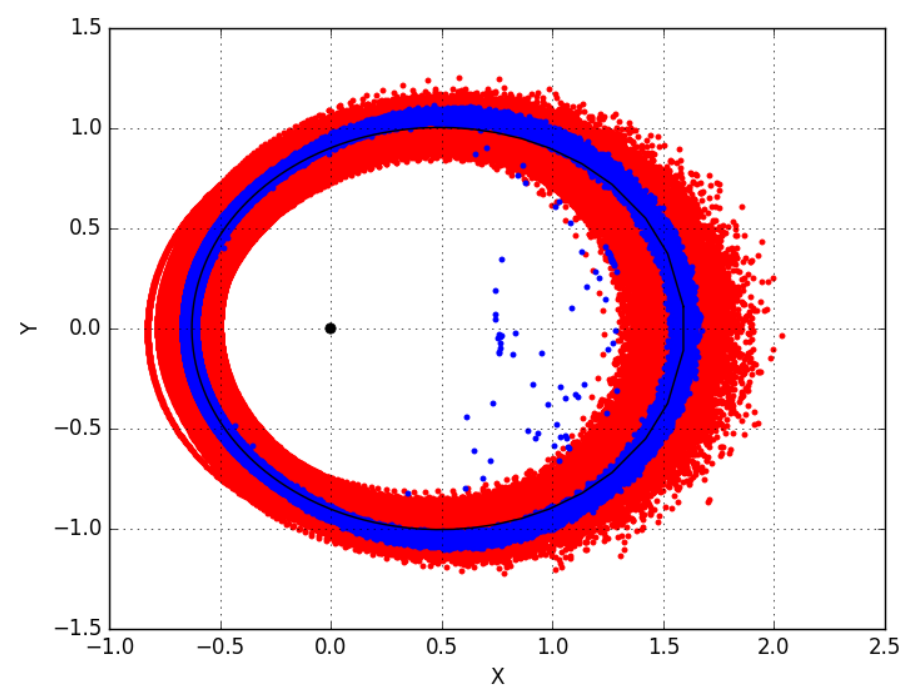

Figure 2. The extent of a bushfire started at the origin as computed by 5000 realisations of the ensemble (blue dots) and the fully stochastic model (red dots). Each of the blue dots represent the position of a single point on the fire line after 1 hour evolution from a single simulation. The solid line is fire line according to the deterministic model with a constant westerly wind of $\underset{\sim}{\mathbf{w}}=(20,0)$.

Figure 2 illustrates the difference between ensemble simulations (blue dots) and stochastic (red dots) simulations. Once again the constant westerly wind result is shown by the solid black line. What can be clearly seen is that the stochastic model produced a significantly greater a mount of variability than that of the ensemble approach to the wind fluctuations. The stochastic model produces, not only a broader range in the possible extent of the head fire after one hour of elapsed time, but also in the back and fl anks. This suggests that an ensemble methodology could potentially grossly underestimate how far a fire will s pread. The ramifications of this in an operational bush fire simulation could range from inefficient al location of re sources to loss of property and lives.

In order to illustrate the differences in the potential propagation of a fire burning according to the two different modelling approaches, we take the final fire line, after 1 hour evolution, from every realisation (ensemble and stochastic respectively). We then scan through a grid of 500 points and test if a point is within the final fire line - i.e. does the point represent a position that was burnt by that particular simulated fire. We repeat the process for every realisation counting the number of times $0 \leq \beta \leq N$ that a given point has been burnt. This allows us to produce a contour outside of which the points are burnt with a probability less than a specified amount. In Figure 3 we see the contours beyond which points are burnt with probability $\frac{\beta}{N} \leq 0.01$ for the ensemble approach (dotted line) and the stochastic model (solid line). The extent of the fire calculated by the stochastic model is clearly greater in all directions, and most notably at the head of the fire. What might be deemed a practically safe point by the ensemble method could be wholly within the danger zone as predicted by the stochastic model.

\section{SUMMARY AND FUTURE WORK}

From their analysis of prediction models, Cruz and Alexander (2014) indicate that there are significant differences in simulated and observed fire spread. Fire spread models are typically deterministic in nature, and as the uncertainty in environmental parameters is not generally accounted for, this leads to large discrepancies between simulated results and the historical bushfire the models are trying to reproduce. Cruz and Alexander discovered that only about three percent (i.e. 35 out of 1278) of predictive models were able to produce the expected results. 
H H. Zazali, et al. Incorporating environmental uncertainty in fire spread modelling

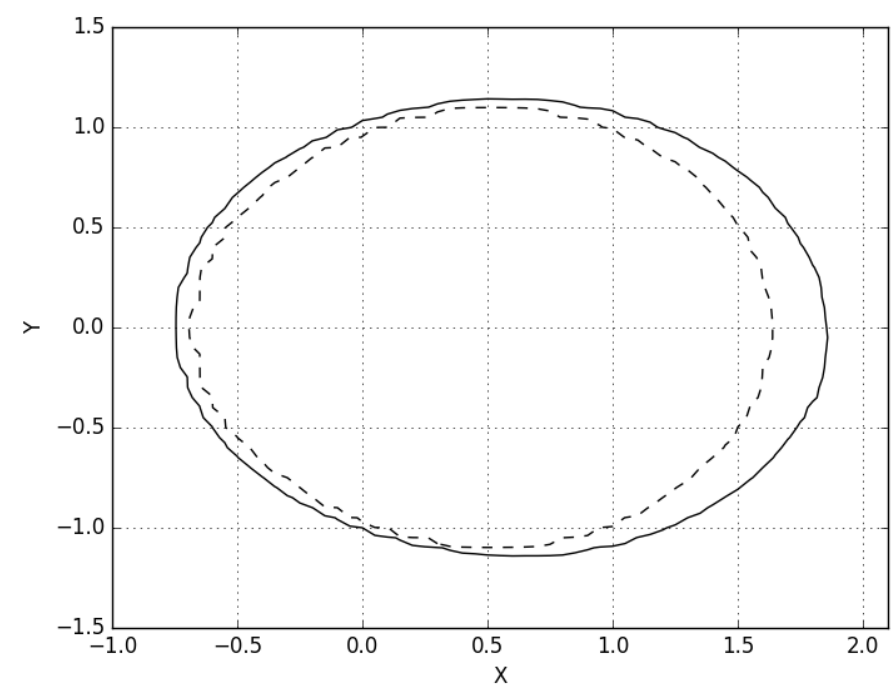

Figure 3. Contours showing regions burnt by $99 \%$ of the simulated fires after 1 hour of simulation time. The solid line is the result for the stochastic model, while the dashed line is for the ensemble approach. The results indicate that fires simulated using the stochastic approach burnt regions $18.2 \%$ further downwind of the origin than those simulated using the ensemble method.

Current ensemble fire spread calculations switch the aim from determining the perimeter of the fire at a particular point in time, to a probabilistic view, in which the output of the ensemble approach provides a measure of the probability of fire impact. To date, however, ensemble models have incorporated environmental variability in an extrinsic way, sampling the input parameters as a separate exercise to the fire propagation modelling.

In contrast, we have considered wind as an example of an environmental feature that should be treated as a stochastic state variable, and compared our stochastic model to a variant of an ensemble of deterministic spread simulations. The stochastic model produced fire lines with much greater variability than the ensemble approach. Furthermore, given our metric $\beta$ of the likelihood that a given point will be burnt, any designated zone of safety is $18.2 \%$ further away from the origin after an hour when calculated by the stochastic model than the ensemble. This has obvious implications if, as we believe, the stochastic approach to the problem of parameter variability is the more realistic of the two considered in the paper.

In addition, we will further investigate the strength of the additive noise $\delta_{1,2}$ in equation (5), by vary them with different position along the fire f ront. This will better reflect the non-stationarity in the variability around the fire perimeter; as, for example, the conditions at the more intense head of the fire, where pyrogenic turbulence is greater, should be expected to be more variable than at the back of the fire.

Moreover, in future work we will change from a front tracking method to the level set paradigm (Sethian, 1996), which performs better in interpreting implicit surfaces evolving around the boundary in environmental inputs. More comparison models with variability of wind data and weather inputs will also be implemented in future work. It is worth noting that implementing stochastic models is not a simple process. Each detail and intricacy needs to be developed properly to ensure the work is successfully delivered. Nevertheless, in our opinion the stochastic model provides more realistic representation of the likely fire spread, and so the extra effort required to implement the stochastic model is justified.

Overall, our results provide a strong indication that the particular way uncertainty is incorporated into fire spread modelling is an important consideration. This provides important insights for future fire spread model development, which will provide fire managers with a greater appreciation of how environmental uncertainty affects their decision making, and a greater capacity to deal with it more effectively.

\section{REFERENCES}

Albini, F. A. (1976). Estimating wildfire behavior and e ffects. General Technical Report, INT - GTR - 30, U.S. Department of Agriculture, Forest Services, Intermountain Forest and Range Experiment Station. 92 
H H. Zazali, et al. Incorporating environmental uncertainty in fire spread modelling

p., Ogden, UT.

Alexander, M. E. and M. G. Cruz (2013). Limitations on the accuracy of model predictions of wildland fire behaviour: A state-of-the-knowledge overview. The Forestry Chronicle 89(3), 372-383.

Anderson, D., E. Catchpole, N. De Mestre, and T. Parkes (1982). Modelling the spread of grass fires. The Journal of the Australian Mathematical Society. Series B. Applied Mathematics 23(04), 451-466.

Boychuk, D., W. J. Braun, R. J. Kulperger, Z. L. Krougly, and D. A. Stanford (2009). A stochastic forest fire growth model. Environmental and Ecological Statistics 16(2), 133-151.

Cruz, M. G. (2010). Monte Carlo-based ensemble method for prediction of grassland fire spread. International Journal of Wildland Fire 19(4), 521-530.

Cruz, M. G. and M. E. Alexander (2014). Uncertainty in model predictions of wildland fire rate of spread. In D. X. Viegas (Ed.), Advances in Forest Fire Research, Coimbra, pp. 466-477. University Press, Coimbra, Portugal.

French, I., R. Cechet, T. Yang, and L. Sanabria (2013). FireDST: Fire Impact and Risk Evaluation Decision Support Tool-Model Description. In 20th International Congress on Modelling and Simulation (MODSIM 2013), Adelaide, Australia.

Fujioka, F. M. (2001). A new methodology for the analysis of two-dimensional fire spread simulations. Fire Meteorology Research Unit. USDA Forest Services. Riverside, CA.

Hajian, M., E. Melachrinoudis, and P. Kubat (2016). Modeling wildfire propagation with the stochastic shortest path: A fast simulation approach. Environmental Modelling \& Software 82, 73-88.

Han, L. and J. W. Braun (2014). Dionysus: a stochastic fire growth scenario generator. Environmetrics 25(6), 431-442.

Johnston, P., G. Milne, and D. Klemitz (2005). Overview of bushfire spread simulation systems. Bushfire CRC Project B6.3.

McArthur, A. G. (1967). Fire behaviour in eucalypt forests. Forest Research Institute, Forest and Timber Bureau of Australia, Leaflet no. 107.

Noble, I., A. Gill, and G. Bary (1980). McArthur's fire-danger meters expressed as equations. Austral Ecology 5(2), 201-203.

Øksendal, B. (1998). Stochastic differential equations: an introduction with applications. 5th ed. Springer Berlin.

Roberts, A. (2012). Modify the improved euler scheme to integrate stochastic differential equations. Technical report. preprint arXiv:1210.0933.

Roberts, S. (1993). A line element algorithm for curve flow problems in the plane. Journal of the Australian Mathematical Society 35, 244-261.

Sethian, J. A. (1996). Level set methods : evolving interfaces in geometry, fluid mechanics, computer vision, and materials science. Cambridge monographs on applied \& computational mathematics. Cambridge: Cambridge University Press.

Shampine, L. F. and M. W. Reichelt (1997). The Matlab ODE suite. SIAM Journal on Scientific Computing $18(1), 1-22$.

Tryggvason, G., B. Bunner, A. Esmaeeli, D. Juric, N. Al-Rawahi, W. Tauber, J. Han, S. Nas, and Y.-J. Jan (2001). A front-tracking method for the computations of multiphase flow. Journal of Computational Physics 169(2), $708-759$.

Twomey, B. and A. Sturgess (2016). Simulation Analysis-based Risk Evaluation (SABRE) Fire: operational stochastic fire spread decision support capability in the Queensland fire and emergency services. Technical report, Brisbane, Australia.

Zhang, Y., S. Lim, and J. J. Sharples (2016). Modelling spatial patterns of wildfire occurrence in South-Eastern Australia. Geomatics, Natural Hazards and Risk 7(6), 1800-1815. 